\title{
Integral equation methods for vesicle electrohydrodynamics in three dimensions
}

\author{
Shravan Veerapaneni*
}

\begin{abstract}
In this paper, we develop a new boundary integral equation formulation that describes the coupled electro- and hydro-dynamics of a vesicle suspended in a viscous fluid and subjected to external flow and electric fields. The dynamics of the vesicle are characterized by a competition between the elastic, electric and viscous forces on its membrane. The classical Taylor-Melcher leaky-dielectric model is employed for the electric response of the vesicle and the Helfrich energy model combined with local inextensibility is employed for its elastic response. The coupled governing equations for the vesicle position and its transmembrane electric potential are solved using a numerical method that is spectrally accurate in space and first-order in time. The method uses a semi-implicit time-stepping scheme to overcome the numerical stiffness associated with the governing equations.
\end{abstract}

\section{Introduction}

Electric-field induced dynamics of soft or deformable particulate suspensions is a fundamental physical phenomenon that arises ubiquitously in natural and engineered systems. Unlike the electrohydrodynamics (EHD) of colloidal suspensions, which received much attention, little progress has been made in direct numerical simulations of the EHD of soft particle suspensions. This is owing to the numerous computational challenges associated with the complex moving geometries and the multi-scale, multi-physics nature of the problem. In this paper, we consider the EHD of a particular soft particle, namely, a vesicle - closed lipid bilayer membrane that encloses a viscous fluid. Understanding vesicle EHD can bring valuable insights into the behavior of general biological cells under applied electric fields since both share the same structural component, the enclosing bilipid membrane. Not surprisingly, characterizing the combined effect of flow and electric fields via experiments on the so called giant unilamellar vesicles is an active area of research $[1,8,29,38,39,42]$.

The vesicle membrane resists bending and is locally inextensible. The Helfrich energy is typically used to model the membrane elastic energy combined with tension as a Lagrange multiplier to enforce the local inextensibility [16, 23, 51]. The Taylor-Melcher model [30, 43, 47], developed in the context of fluid-fluid interfaces, has been extended to model vesicle EHD in [53]. In this model, the electric charge convection is neglected and the charges are assumed to be present only at the interface and not in the bulk. Unlike simple interfaces, the vesicle membrane acts as a charging capacitor when an external electric field is applied since it is impermeable to ions.

Theoretical investigation of vesicle EHD has been done only recently in [45, 53]. Using small deformation theory, they were able to obtain the experimentally observed prolate-to-oblate shape

*Department of Mathematics, University of Michigan, Ann Arbor, MI 48109, shravan@umich.edu. 
transitions [1] that depend on the interior-to-exterior fluid conductivity ratio (see [52] for a review on the dynamics of vesicles in electric fields). A spheroidal model has been developed in [33, 55] that can handle large deformations (as long as the shape remains spheroidal). While the aforementioned models offered key insights in some settings, they cannot, however, be applied to general three-dimensional EHD flows. Methods for direct numerical simulations, therefore, need to be developed to handle the large membrane deformations and dynamics in general 3D flows. They also are crucial for technological applications, specially those classified by electroporation $[7,13,17,32]$, electromanipulation [18], and electroformation [48], which require precise knowledge of the membrane variables such as the tension and transmembrane electric potential to predict their efficacy.

Numerical methods for solving the coupled electric, elastic and hydrodynamic governing equations for the vesicle EHD have been developed only recently [19-21, 27-29]. While the works of [20] and [21] use the immersed interface method (IIM) to solve the electric potential problem and level sets to track the moving interface, the recent work of [19] employs a hybrid approach and uses immersed boundary method for fluid flow and IIM to evolve the electric variables. On the other hand, the works of [27] and [29] are based on boundary integral equation (BIE) methods, which are particularly well-suited for the vesicle EHD problem since the governing equations for the fluid motion as well as the electric potential are linear. In this setting, BIE methods offer several advantages over domain discretization methods as they lead to reduction in dimensionality, satisfy the far-field boundary conditions exactly and can be solved via highly scalable fast algorithms.

However, both [27] and [29] were restricted to two-dimensional problems. Several challenges confront the design of BIE methods for three dimensional EHD problem including (i) the interfacial conditions for the electric problem, treating the membrane as a charging capacitor, lead to firstkind integral equations when the standard direct formulation is used (as was done in [27, 29]), (ii) the high-order spatial derivatives in the elastic force arising from the Helfrich energy introduce numerical stiffness into the interfacial evolution equation, (iii) due to lack of in-plane shear resistance in the model, numerical instabilities arise because of the loss of mesh (or surface representation) quality as the vesicle undergoes large deformations. Some of the challenges have been addressed in our previous work on vesicle hydrodynamics [51]. The present work extends [51] to the EHD setting.

Contributions. We employ a new indirect formulation and derive second-kind BIEs for solving the electric potential problem. Combined with the interfacial evolution, we arrive at a coupled system of integro-differential equations that govern the vesicle EHD. We introduce a semi-implicit time-stepping scheme to solve this coupled system and evolve the EHD variables and the membrane position. We construct a spectrally-accurate scheme to compute the interfacial forces, differential and integral operators on the interface using spherical harmonic representations. We outline a spectrally-accurate numerical method to compute the hyper-singular integrals that arise in our BIE formulation. It is based on reducing them to weakly singular integrals and using a fast polerotation based quadrature scheme [12]. We present numerical results verifying the convergence of our method and simulations that qualitatively match the existing experimental and theoretical results.

The paper is organized as follows. In the next section, we describe the partial differential equations governing the fluid motion and the electric potential along with the interfacial conditions. In Section 3, we reformulate the governing equations as integro-differential equations with 
the unknowns residing only on the vesicle membrane. In Section 4, we introduce our spatial discretization and time-marching scheme to evolve the membrane position and the electric variables. Subsequently, we present numerical results testing the accuracy and stability of our method and simulations in Section 5, followed by conclusions and future directions in Section 6 .

\section{Problem formulation}

Consider a vesicle suspended in an unbounded viscous fluid domain, subjected to an imposed flow $\mathbf{v}_{\infty}(\mathbf{x})$, for any $\mathbf{x} \in \mathbb{R}^{3}$. Assume that the interior and exterior fluids have the same viscosity $\mu$ and the same dielectric permittivity $\epsilon$ while their conductivities differ, given by $\sigma_{i}$ and $\sigma_{e}$, respectively. Let $\mathbf{x}$ be the position of the vesicle membrane $\gamma, \mathbf{v}$ the fluid velocity and $p$ the pressure. In the vanishing Reynolds number limit, the governing equations for the vesicle hydrodynamics can be written as:

$$
\begin{array}{rlll}
-\nabla p+\mu \triangle \mathbf{v}=0 \quad \text { in } \quad \mathbb{R}^{3} \backslash \gamma & \text { (conservation of momentum in bulk fluid) } \\
\nabla \cdot \mathbf{v}=0 \quad \text { in } \quad \mathbb{R}^{3} \backslash \gamma & \text { (fluid incompressibility) } \\
\mathbf{v}(\mathbf{x}) \rightarrow \mathbf{v}_{\infty}(\mathbf{x}) \quad \text { as } \quad\|\mathbf{x}\| \rightarrow \infty & \text { (far-field boundary condition) } \\
\dot{\mathbf{x}}=\mathbf{v} \quad \text { on } \quad \gamma & \text { (velocity continuity), } \\
\nabla_{\gamma} \cdot \dot{\mathbf{x}}=0 & \text { (surface inextensibility) } \\
{\left[\left[\mathbf{n} \cdot\left(\Sigma^{e l}+\Sigma^{h d}\right)\right]\right]_{\gamma}=\mathbf{f}_{m}} & \text { (membrane force balance) }
\end{array}
$$

In the last equation, $\llbracket \cdot \rrbracket_{\gamma}$ denotes the jump across the interface (e.g., $\llbracket \sigma \rrbracket_{\gamma}=\sigma_{i}-\sigma_{e}$ ), $\mathbf{n}$ is the outward normal to $\gamma, \Sigma^{e l}$ is the electric stress, $\Sigma^{h d}$ is the hydrodynamic stress and $\mathbf{f}_{m}$ is the total membrane force. The classical Helfrich energy model for the vesicle membrane and an augmented Lagrangian approach to enforce the surface inextensibility locally lead to a bending force $\mathbf{f}_{b}$ and a tension force $\mathbf{f}_{\lambda}$ on the membrane, so that $\mathbf{f}_{m}=\mathbf{f}_{b}+\mathbf{f}_{\lambda}$. They are defined by [51],

$$
\mathbf{f}_{b}=-\kappa_{B}\left(\triangle_{\gamma} H+2 H\left(H^{2}-K\right)\right) \mathbf{n}, \quad \mathbf{f}_{\lambda}=\lambda \triangle_{\gamma} \mathbf{x}+\nabla_{\gamma} \lambda
$$

where $\kappa_{B}$ is the bending modulus, $H$ is the mean curvature and $K$ is the Gaussian curvature. The tension $\lambda$ acts as a Lagrange multiplier to enforce the surface inextensibility constraint and it is computed as part of the solution.

The electric stress $\Sigma^{e l}$ is given by the Maxwell stress tensor, defined as,

$$
\Sigma^{e l}=\epsilon \mathbf{E} \otimes \mathbf{E}-\frac{1}{2} \epsilon\|\mathbf{E}\|^{2} \mathbf{I}
$$

Therefore, the electric field $\mathbf{E}$ on both sides of the vesicle membrane needs to be determined to enforce the stress balance at the interface for a given vesicle shape. We use the Taylor-Melcher leaky dielectric model [30], in which, the electric charges are assumed to be present only at the interface and not in the bulk. Hence, the electric field is solenoidal in the bulk and the electric potential $\phi$ satisfies the Laplace equation. Assuming that the vesicle membrane is charge-free and has a conductivity $G_{m}$, a capacitance $C_{m}$, the boundary value problem for the electric potential 
can be written as [44]:

$$
\begin{array}{rll}
-\triangle \phi=0 \quad \text { in } \quad \mathbb{R}^{3} \backslash \gamma & \text { (potential equation), } \\
\llbracket \mathbf{n} \cdot(\sigma \nabla \phi) \rrbracket_{\gamma}=0 & \text { (current continuity), } \\
\llbracket \phi \rrbracket_{\gamma}=V_{m} & \text { (transmembrane potential), } \\
-\nabla \phi(\mathbf{x}) \rightarrow \mathbf{E}_{\infty}(\mathbf{x}) \quad \text { as } \quad\|\mathbf{x}\| \rightarrow \infty & \text { (far-field boundary condition), } \\
C_{m} \dot{V}_{m}+G_{m} V_{m}=-\mathbf{n} \cdot\left(\sigma_{i} \nabla \phi_{i}\right) \quad \text { on } \gamma & \text { (conservation of electric current). }
\end{array}
$$

Note that we have neglected the charge convection due to fluid flow along the membrane while enforcing the current continuity in the direction normal to $\gamma$. The jump in the electric potential across the membrane, $V_{m}$, also termed as the transmembrane potential, is an unknown that needs to be determined as part of the solution process. In the current conservation equation (4e), $\phi_{i}$ denotes the interior electric potential evaluated at the interface (analogously $\phi_{e}$, the exterior potential). $C_{m} \dot{V}_{m}$ is the transient current due to charging of the capacitative interface (the vesicle membrane).

When an external electric field is applied, charges accumulate on both sides of the membrane, giving rise to a non-zero transmembrane potential. Since the electric field is discontinuous across the membrane, it experiences an electric stress (3). The vesicle deforms until the elastic stress due to bending and tension balances out this electric stress and the hydrodynamic stress at the interface.

\section{Integral equation formulation}

Boundary integral equation formulation for vesicle hydrodynamics is now well-established and several studies have employed it for problems in two $[3,5,6,26,36,50]$ and three $[4,10,24,37,46,51]$ dimensions. The standard procedure, for a single vesicle with no viscosity-contrast, is to convert the PDE formulation (1) into coupled integro-differential equations of the following form [51]:

$$
\dot{\mathbf{x}}=\mathbf{v}_{\infty}(\mathbf{x})+\int_{\gamma} G_{s}(\mathbf{x}-\mathbf{y}) \mathbf{f}(\mathbf{y}) d \gamma, \quad \nabla_{\gamma} \cdot \dot{\mathbf{x}}=0,
$$

where $\mathbf{x}$ is the membrane position at certain time, $\mathbf{f}$ is the total force exerted by the membrane on the fluid, given by $\mathbf{f}=\left[\left[\mathbf{n} \cdot \Sigma^{h d}\right]\right]_{\gamma}$, and $G_{s}$ is the Stokesian fundamental solution given by,

$$
G_{s}(\mathbf{x}-\mathbf{y})=\frac{1}{8 \pi \mu}\left(\frac{1}{\|\mathbf{x}-\mathbf{y}\|} \mathbf{I}+\frac{(\mathbf{x}-\mathbf{y}) \otimes(\mathbf{x}-\mathbf{y})}{\|\mathbf{x}-\mathbf{y}\|^{3}}\right) .
$$

In the absence of electric fields, $\mathbf{f}$ is simply the sum of bending and tension forces and the two equations in (5) are numerically solved for the two unknowns, the tension $\lambda$, and the membrane position update, at any given time-step. Classical results such as the existence of various families of equilibrium shapes in quiescent flows and tank-treading of a vesicle suspended in linear shear flows can be obtained from the numerical solution of these integro-differential equations.

In the presence of electric fields, however, $\mathbf{f}=\mathbf{f}_{m}-\left[\left[\mathbf{n} \cdot \Sigma^{e l}\right]\right]_{\gamma}$, and the electric stress on the membrane needs to computed by solving (4) for a given vesicle shape. Since (4) is a linear partial differential equation, similar to the fluid problem, we can recast it as a boundary integral equation with the unknowns residing only on the interface. We discuss this formulation next. 


\subsection{BIE formulation for electric potential}

Similar to (5), our goal is to express the solution to (4) in terms of operators defined on the boundary $\gamma$ only. BIEs for (4) can be formulated in multiple ways (including the standard direct BIE formulation [27]), however, the main objective is to arrive at integral equations that are wellconditioned. Towards this end, we represent the solution to (4) as [54],

$$
\phi(\mathbf{x})=-\mathbf{E}_{\infty} \cdot \mathbf{x}+\mathcal{S}[q](\mathbf{x})-\mathcal{D}\left[V_{m}\right](\mathbf{x})
$$

where the membrane charge density, $q=\llbracket \partial \phi / \partial n \rrbracket_{\gamma}$ and the Laplace single and double layer integral operators, $\mathcal{S}[\cdot]$ and $\mathcal{D}[\cdot]$ respectively, are defined by

$$
\begin{gathered}
\mathcal{S}[f](\mathbf{x})=\int_{\gamma} G(\mathbf{x}-\mathbf{y}) f(\mathbf{y}) d \gamma(\mathbf{y}), \quad \mathcal{D}[f](\mathbf{x})=\int_{\gamma} \frac{\partial G(\mathbf{x}-\mathbf{y})}{\partial \mathbf{n}(\mathbf{y})} f(\mathbf{y}) d \gamma(\mathbf{y}), \\
\text { where } \quad G(\mathbf{x}-\mathbf{y})=\frac{1}{4 \pi\|\mathbf{x}-\mathbf{y}\|} \quad \text { and } \quad \frac{\partial G(\mathbf{x}-\mathbf{y})}{\partial \mathbf{n}(\mathbf{y})}=\frac{1}{4 \pi} \frac{(\mathbf{x}-\mathbf{y}) \cdot \mathbf{n}}{\|\mathbf{x}-\mathbf{y}\|^{3}}
\end{gathered}
$$

The representation (7) satisfies equations (4a, 4c, 4d) automatically due to the fact that $G$ and its derivatives are fundamental solutions of the Laplace equation and that the single-layer operator is continuous across $\gamma$. Enforcing the remaining interfacial conditions (4b, 4e) will give us two BIEs to solve for the two unknowns $q$ and $V_{m}$. To derive these integral equations, we introduce the derivatives of the single and double layer integral operators $\mathcal{S}^{\prime}[\cdot]$ and $\mathcal{D}^{\prime}[\cdot]$ defined as,

$$
\mathcal{S}^{\prime}[f](\mathbf{x})=\frac{\partial}{\partial \mathbf{n}(\mathbf{x})} \int_{\gamma} G(\mathbf{x}-\mathbf{y}) f(\mathbf{y}) d \gamma(\mathbf{y}), \quad \mathcal{D}^{\prime}[f](\mathbf{x})=\frac{\partial}{\partial \mathbf{n}(\mathbf{x})} \int_{\gamma} \frac{\partial G(\mathbf{x}-\mathbf{y})}{\partial \mathbf{n}(\mathbf{y})} f(\mathbf{y}) d \gamma(\mathbf{y}) .
$$

The interior and exterior limits of the electric potential evaluated at the membrane, denoted by $\left\{\phi_{i}, \phi_{e}\right\}$ respectively, and their normal derivatives, $\left\{\partial \phi_{i} / \partial \mathbf{n}, \partial \phi_{e} / \partial \mathbf{n}\right\}$, can be derived using the standard jump conditions for the layer potentials and their normal derivatives [25] as:

$$
\begin{array}{r}
\phi_{i}(\mathbf{x})=-\mathbf{E}_{\infty} \cdot \mathbf{x}+\mathcal{S}[q](\mathbf{x})+\frac{1}{2} V_{m}(\mathbf{x})-\mathcal{D}\left[V_{m}\right](\mathbf{x}), \\
\phi_{e}(\mathbf{x})=-\mathbf{E}_{\infty} \cdot \mathbf{x}+\mathcal{S}[q](\mathbf{x})-\frac{1}{2} V_{m}(\mathbf{x})-\mathcal{D}\left[V_{m}\right](\mathbf{x}), \\
\frac{\partial \phi_{i}}{\partial \mathbf{n}}(\mathbf{x})=-\mathbf{E}_{\infty} \cdot \mathbf{n}(\mathbf{x})+\frac{1}{2} q(\mathbf{x})+\mathcal{S}^{\prime}[q](\mathbf{x})-\mathcal{D}^{\prime}\left[V_{m}\right](\mathbf{x}), \\
\frac{\partial \phi_{e}}{\partial \mathbf{n}}(\mathbf{x})=-\mathbf{E}_{\infty} \cdot \mathbf{n}(\mathbf{x})-\frac{1}{2} q(\mathbf{x})+\mathcal{S}^{\prime}[q](\mathbf{x})-\mathcal{D}^{\prime}\left[V_{m}\right](\mathbf{x}) .
\end{array}
$$

where $\eta=\left(\sigma_{i}-\sigma_{e}\right) /\left(\sigma_{i}+\sigma_{e}\right)$. Substituting (11c, 11d) in the interfacial condition (4b), we obtain the following boundary integral equation for the unknown $q$ :

$$
\left(\frac{1}{2}+\eta \mathcal{S}^{\prime}\right) q=\eta \mathbf{E}_{\infty} \cdot \mathbf{n}+\eta \mathcal{D}^{\prime}\left[V_{m}\right]
$$

The advantage of the representation (7) is now clear: (12) is a Fredholm integral equation of the second-kind, which can be solved rapidly using iterative methods. To obtain one more equation for the unknown $V_{m}$, we need to determine the normal derivative of the electric potential (from (4e)). 
We can express the normal derivative in terms of $q$ by solving the linear equations $\llbracket \partial \phi / \partial \mathbf{n} \rrbracket_{\gamma}=q$ and $\llbracket \sigma \partial \phi / \partial \mathbf{n} \rrbracket_{\gamma}=0$. The result is:

$$
\frac{\partial \phi_{i}}{\partial \mathbf{n}}=\frac{\sigma_{e}}{\sigma_{e}-\sigma_{i}} q, \quad \frac{\partial \phi_{e}}{\partial \mathbf{n}}=\frac{\sigma_{i}}{\sigma_{e}-\sigma_{i}} q .
$$

Substituting this in (4e), we obtain the following integro-differential equation for $V_{m}$ evolution:

$$
C_{m} \dot{V}_{m}+G_{m} V_{m}=\frac{\sigma_{i} \sigma_{e}}{\sigma_{i}+\sigma_{e}}\left(\frac{1}{2}+\eta \mathcal{S}^{\prime}\right)^{-1}\left(\mathbf{E}_{\infty} \cdot \mathbf{n}+\mathcal{D}^{\prime}\left[V_{m}\right]\right)
$$

Therefore, given the vesicle shape, the transmembrane potential can be evaluated independently by solving (14). Knowing $q$ and $V_{m}$, we can evaluate the electric potential at any point in the interior or exterior of $\gamma$ using (7). Finally, we need to evaluate the jump in the Maxwell stress tensor (3) to determine the electric force on the membrane. To do so, we first compute the electric potential near the membrane and its normal derivatives using (11). Then, the interior electric field near the membrane $\mathbf{E}_{i}$ (similarly, $\mathbf{E}_{e}$ ) is determined using the expression,

$$
\mathbf{E}_{i}=-\nabla_{\gamma} \phi_{i}-\frac{\partial \phi_{i}}{\partial \mathbf{n}} \mathbf{n}
$$

In summary, the PDE formulation governing the vesicle $\operatorname{EHD}(1,4)$ has been reduced to a BIE formulation in the form of coupled evolution equations for the membrane position (5) and the transmembrane potential (14). The details of the numerical implementation are discussed next.

\section{Numerical Method}

In this section, we discuss methods for the numerical solution of the coupled integro-differential equations governing the vesicle EHD. For the most part, we follow the general numerical framework for three-dimensional vesicle flows introduced in our previous work [51].

\subsection{Spatial discretization}

We use spherical harmonic approximations to numerically represent the vesicle membrane and the interfacial forces. The electric charge density on the surface, for instance, is approximated by its truncated spherical harmonic expansion of degree $p$ :

$$
\begin{gathered}
q(\theta, \phi)=\sum_{n=0}^{p} \sum_{m=-n}^{n} q_{n}^{m} Y_{n}^{m}(\theta, \phi), \\
\theta \in[0, \pi], \quad \phi \in[0,2 \pi] .
\end{gathered}
$$

Here, $\theta$ is the polar angle, $\phi$ is the azimuthal angle, $q_{n}^{m}$ are the spherical harmonic coefficients of $q$, and $Y_{n}^{m}$ is a spherical harmonic of degree $n$ and order $m$ defined, in terms of the associated Legendre functions $P_{n}^{m}[40]$, by

$$
Y_{n}^{m}(\theta, \phi)=\sqrt{\frac{2 n+1}{4 \pi}} \sqrt{\frac{(n-|m|) !}{(n+|m|) !}} P_{n}^{|m|}(\cos \theta) e^{i m \phi} .
$$


The finite-term spherical harmonic approximation, such as (16), is superalgebraically convergent with $p$ for smooth functions. The forward and inverse spherical harmonic transforms can be used to switch from physical to spectral domain, $q \rightarrow q_{n}^{m}$, and vice-versa. A standard choice for the numerical integration scheme required for computing these transforms is to use the trapezoidal rule in the azimuthal direction and the Gauss-Legendre quadrature in the polar direction. The resulting grid points in the parametric domain are given by

$$
\left\{\theta_{j}=\cos ^{-1}\left(t_{j}\right), j=0, \ldots p\right\}, \quad \text { and } \quad\left\{\phi_{k}=\frac{2 \pi k}{2 p+2}, k=0, \ldots, 2 p+1\right\},
$$

where $t_{j}$ 's are the nodes of the $(p+1)$-point Gauss-Legendre quadrature on $[-1,1]$.

The differential operators on $\gamma$ are computed via spectral differentiation. For example, the surface gradient of tension is defined in terms of the first fundamental forms by,

$$
\nabla_{\gamma} \sigma=\left(\frac{G \mathbf{x}_{\theta}-F \mathbf{x}_{\phi}}{W^{2}}\right) \sigma_{\theta}+\left(\frac{E \mathbf{x}_{\phi}-F \mathbf{x}_{\theta}}{W^{2}}\right) \sigma_{\phi}
$$

where $E=\mathbf{x}_{\theta} \cdot \mathbf{x}_{\theta}, \quad F=\mathbf{x}_{\theta} \cdot \mathbf{x}_{\phi}, \quad G=\mathbf{x}_{\phi} \cdot \mathbf{x}_{\phi}, \quad W=\sqrt{E G-F^{2}}$.

The $\theta$ and $\phi$ derivatives of $\sigma$ (and similarly that of $\mathbf{x}$ ) at a discrete point $\left(\theta_{j}, \phi_{k}\right)$ are computed using its spherical harmonic coefficients:

$$
\sigma_{\theta}\left(\theta_{j}, \phi_{k}\right)=\sum_{n=0}^{p} \sum_{m=-n}^{n} \sigma_{n}^{m}\left(Y_{n}^{m}\left(\theta_{j}, \phi_{k}\right)\right)_{\theta}, \quad \sigma_{\phi}\left(\theta_{j}, \phi_{k}\right)=\sum_{n=0}^{p} \sum_{m=-n}^{n} \sigma_{n}^{m}\left(Y_{n}^{m}\left(\theta_{j}, \phi_{k}\right)\right)_{\phi} .
$$

The main drawback of this spectrally-accurate differentiation scheme is that it leads to the well-known aliasing phenomena. A standard practice to mitigate this problem is to upsample the functions using spherical harmonic interpolation, compute the derivatives via (21) on the finer grid and then restrict to the original grid $[51,56]$. We follow the same procedure here and use an upsampling factor of two.

Singular integration. The Stokes and the Laplace layer integral operators are weakly singular with their kernels exhibiting a $1 / r$ type of singularity. Therefore, the following spectrally-accurate numerical integration rule for smooth functions on the sphere is not efficient in computing layer potentials:

$$
\int_{\gamma} q(\mathbf{y}) d \gamma(\mathbf{y})=\sum_{j=0}^{p} \sum_{k=0}^{2 p+1} w_{j k} q\left(\mathbf{y}\left(\theta_{j}, \phi_{k}\right)\right) \frac{W\left(\theta_{j}, \phi_{k}\right)}{\sin \theta_{j}}, \quad \text { where } \quad w_{j k}=\frac{2 \pi}{2 p+2} \lambda_{j}
$$

and $\lambda_{j}$ 's are the Gauss-Legendre quadrature weights. In [12], we introduced a fast algorithm for computing the singular integrals which exploits the fact that at the north (or the south) pole of the spherical grid, the area element $W$ vanishes, thereby, making the integrand of the layer potentials non-singular. To evaluate the layer potential at any arbitrary location $\mathbf{x}$ on the surface $\gamma$, the coordinate system is rotated so that $\mathbf{x}$ coincides with the north pole. At the north pole $\mathbf{x}(0,0)$, the following quadrature rule for computing $\mathcal{S}[q]$ is spectrally-accurate $[11,12,14,51]$ :

$$
\frac{1}{4 \pi} \int_{\gamma} \frac{q(\mathbf{y})}{\|\mathbf{x}(0,0)-\mathbf{y}\|} d \gamma(\mathbf{y})=\sum_{j=0}^{p}\left(\frac{\sum_{n=0}^{p} P_{n}\left(\cos \theta_{j}\right)}{\cos \left(\theta_{j} / 2\right)}\right) \sum_{k=0}^{2 p+1} \frac{w_{j k} q\left(\mathbf{y}\left(\theta_{j}, \phi_{k}\right)\right) W\left(\theta_{j}, \phi_{k}\right)}{\left\|\mathbf{x}(0,0)-\mathbf{y}\left(\theta_{j}, \phi_{k}\right)\right\|}
$$


The same kind of quadrature rule can be applied in computing the Laplace double layer potential and the Stokes single-layer potential [51], required for the vesicle EHD simulation. However, this scheme is not efficient in computing the derivative of the double-layer potential since the kernel of the integral operator $\mathcal{D}^{\prime}[\cdot]$ is hyper-singular. We discuss a modified scheme next.

\subsection{Derivative of the double layer potential}

Although specialized quadrature rules are required for hyper-singular integrals [22], an alternate strategy can be applied in our setting since derivatives can be computed with spectral accuracy. Lemma 2.2 of [15] (Page 59) transforms the two normal derivatives on the kernel of $\mathcal{D}^{\prime}[\cdot]$ into one tangential derivative on the density and one on the potential using integration by parts, e.g.,

$$
\begin{aligned}
\mathcal{D}^{\prime}\left[V_{m}\right](\mathbf{x})=\frac{\partial}{\partial \tau_{1}(\mathbf{x})} \int_{\gamma} G(\mathbf{x}, \mathbf{y}) \frac{\partial V_{m}(\mathbf{y})}{\partial \tau_{1}(\mathbf{y})} d \gamma(\mathbf{y}) & +\frac{\partial}{\partial \tau_{2}(\mathbf{x})} \int_{\gamma} G(\mathbf{x}, \mathbf{y}) \frac{\partial V_{m}(\mathbf{y})}{\partial \tau_{2}(\mathbf{y})} d \gamma(\mathbf{y}) \\
& +\frac{\partial}{\partial \tau_{3}(\mathbf{x})} \int_{\gamma} G(\mathbf{x}, \mathbf{y}) \frac{\partial V_{m}(\mathbf{y})}{\partial \tau_{3}(\mathbf{y})} d \gamma(\mathbf{y})
\end{aligned}
$$

where the vectors $\tau_{1}(\mathbf{x}), \tau_{2}(\mathbf{x})$ and $\tau_{3}(\mathbf{x})$ reside in the tangent plane at the point $\mathbf{x}$ on the surface and are defined in terms of the components of normal vector as,

$$
\tau_{1}(\mathbf{x})=\left(0, n_{3}(\mathbf{x}),-n_{2}(\mathbf{x})\right), \quad \tau_{2}(\mathbf{x})=\left(-n_{3}(\mathbf{x}), 0, n_{1}(\mathbf{x})\right), \quad \tau_{3}(\mathbf{x})=\left(n_{2}(\mathbf{x}),-n_{1}(\mathbf{x}), 0\right) .
$$

The tangential derivatives of the scalar function $V_{m}$ on the surface can simply be computed from its surface gradient (defined in (19)), for example,

$$
\frac{\partial V_{m}(\mathbf{y})}{\partial \tau_{1}(\mathbf{y})}=\tau_{1}(\mathbf{y}) \cdot \nabla_{\gamma} V_{m}(\mathbf{y})
$$

Similarly the tangential derivative of the single-layer potential, $\partial / \partial \tau_{\mathbf{x}} \mathcal{S}[\cdot]$, can be computed from its surface gradient.

\subsection{Time-stepping}

One of the main difficulties associated with simulating vesicle flows, compared to other particulate flows, is that the interfacial forces are highly nonlinear and sustain fourth-order derivatives in the interfacial position. Consequently, the evolution equation (5) is numerically stiff and the explicit time-marching schemes tend to be prohibitively expensive because of the stringent stability restrictions on the time-step size. In [37, 49-51], we developed semi-implicit time-stepping schemes for two- and three-dimensional vesicle flows that overcome the numerical stiffness with only a modest increase in cost per time-step compared to fully explicit schemes. McConnell et al. [27] extended these schemes to study vesicle EHD in two dimensions by treating the electric force explicitly. In this work too, we treat the electric force on the membrane explicitly so as to decouple the timemarching schemes for membrane position (5) and transmembrane potential (14) evolutions. Both evolution equations are then solved using semi-implicit schemes.

Given the location of marker points $\left\{\mathbf{x}^{n}\left(\theta_{j}, \phi_{k}\right), j=0, \ldots p, k=0, \ldots, 2 p+1\right\}$ on the vesicle membrane at time $n \triangle t$ and the electric force on it, denoted by $\mathbf{f}_{E}^{n}$, we use the following first-order 
time-stepping scheme to discretize the evolution equation (5) and compute $\mathbf{x}^{n+1}$ :

$$
\begin{gathered}
\frac{\mathbf{x}^{n+1}-\mathbf{x}^{n}}{\Delta t}=\mathbf{v}_{\infty}\left(\mathbf{x}^{n}\right)+\int_{\gamma} G_{s}\left(\mathbf{x}^{n}-\mathbf{y}^{n}\right)\left(\mathbf{f}_{b}^{n+1}+\mathbf{f}_{\lambda}^{n+1}-\mathbf{f}_{E}^{n}\right) d \gamma\left(\mathbf{y}^{n}\right), \\
\nabla_{\gamma} \cdot\left(\mathbf{v}_{\infty}\left(\mathbf{x}^{n}\right)+\int_{\gamma} G_{s}\left(\mathbf{x}^{n}-\mathbf{y}^{n}\right)\left(\mathbf{f}_{b}^{n+1}+\mathbf{f}_{\lambda}^{n+1}-\mathbf{f}_{E}^{n}\right) d \gamma\left(\mathbf{y}^{n}\right)\right)=0 .
\end{gathered}
$$

In this semi-implicit scheme, a suitable linearization for the nonlinear membrane forces, $\mathbf{f}_{b}^{n+1}$ and $\mathbf{f}_{\lambda}^{n+1}$, must be found that potentially overcomes the numerical stiffness. A natural recipe is to treat the terms with highest-order spatial derivatives implicitly and the rest explicitly. Suppressing the superscripts on explicitly treated terms to simplify the notation, our choice for the membrane forces at $(n+1) \triangle t$ is given by [51]:

$$
\begin{array}{r}
\mathbf{f}_{b}^{n+1}=-\left(\Delta_{\gamma} H^{n+1}+2 H^{n+1}\left(H^{2}-K\right)\right) \mathbf{n}, \\
\text { where } H^{n+1}=\frac{1}{2 W^{2}}\left(E \mathbf{x}_{v v}^{n+1}-2 F \mathbf{x}_{u v}^{n+1}+G \mathbf{x}_{u u}^{n+1}\right) \cdot \mathbf{n}, \\
\mathbf{f}_{\lambda}^{n+1}=\lambda^{n+1} \Delta_{\gamma} \mathbf{x}+\nabla_{\gamma} \lambda^{n+1} .
\end{array}
$$

Plugging (28) into (27), we get two linear equations for the two unknowns $\mathbf{x}^{n+1}$ and $\sigma^{n+1}$. We solve them using the GMRES method [41] combined with a preconditioner developed in [51] based on analytically obtained spectrum of the integro-differential operators in (5) for the special case of the unit sphere. The next step is to update the electric field $\mathbf{f}_{E}^{n}$ to $\mathbf{f}_{E}^{n+1}$. We introduce the operator $\mathcal{L}$ defined by,

$$
\mathcal{L}=\frac{\sigma_{i} \sigma_{e}}{\sigma_{i}+\sigma_{e}}\left(\frac{1}{2}+\eta \mathcal{S}^{\prime}\right)^{-1},
$$

to simplify the description of the algorithm. The main steps involved in advancing $\mathbf{f}_{E}$ from $n \triangle t$ to $(n+1) \triangle t$ can now be summarized as follows.

1. Apply the backward Euler time-stepping scheme on (14) to obtain the following linear system of equations for the unknowns $\left\{V_{m}^{n+1}\left(\theta_{j}, \phi_{k}\right), j=0, \ldots p, k=0, \ldots, 2 p+1\right\}$ :

$$
\left(C_{m}+\triangle t G_{m}-\triangle t \mathcal{L} \mathcal{D}^{\prime}\right) V_{m}^{n+1}=C_{m} V_{m}^{n}+\triangle t \mathcal{L}\left[\mathbf{E}_{\infty} \cdot \mathbf{n}\right] .
$$

Note that the superscripts are again dropped for explicitly treated terms. Use the formula for the DLP derivative (24) and an iterative method (GMRES) to solve (30).

2. Compute the charge density on the membrane $q^{n+1}$ by solving (12),

$$
q^{n+1}=\frac{\sigma_{i}-\sigma_{e}}{\sigma_{i} \sigma_{e}} \mathcal{L}\left[\mathbf{E}_{\infty} \cdot \mathbf{n}+\mathcal{D}^{\prime}\left[V_{m}^{n+1}\right]\right]
$$

3. From $V_{m}^{n+1}$ and $q^{n+1}$, evaluate the boundary data $\left\{\phi_{i}^{n+1}, \phi_{e}^{n+1}, \partial \phi_{i}^{n+1} / \partial \mathbf{n}, \partial \phi_{e}^{n+1} / \partial \mathbf{n}\right\}$ using $(11 \mathrm{a}-\mathrm{b})$ and $(13)$.

4. Evaluate the membrane electric fields in the interior and exterior

$$
\mathbf{E}_{i}=-\nabla_{\gamma} \phi_{i}^{n+1}-\frac{\partial \phi_{i}^{n+1}}{\partial \mathbf{n}} \mathbf{n}, \quad \mathbf{E}_{e}=-\nabla_{\gamma} \phi_{e}^{n+1}-\frac{\partial \phi_{e}^{n+1}}{\partial \mathbf{n}} \mathbf{n}
$$


5. Compute the Maxwell stress tensor (3) on both sides of the membrane and use it to compute $\mathbf{f}_{E}^{n+1}$.

The electric force can then used to advance the membrane position using (27a) in the subsequent time-step. Steps (i) through (v) are repeated for every time-step. This completes the description of our first-order in time and spectrally-accurate in space numerical solver to simulate vesicle EHD. The semi-implicit scheme can be generalized to achieve high-order accuracy in time via backward difference formulas [2] or spectral-deferred correction methods [34]. Our implementation has two additional components not discussed in this paper, namely, anti-aliasing and reparameterization schemes. Both of these algorithms, essential to maintain the quality of numerical representations and thereby to the overall stability of the solver, are described at length in [37] and [51].

\section{Results}

We implemented the numerical algorithm described in Section 4 and here we report its performance on three examples. In the first example, we construct an analytical test case to verify the BIE formulation and the accuracy of the spatial discretization scheme. In the second example, we demonstrate that the experimentally-observed, transient cylindrical shapes strongly depend on the bending modulus of the vesicle membrane. In the last example, we illustrate the tank-treading phenomenon in applied shear flow and electric fields.

Example 1 (Spatial convergence test). First, we consider a test case to verify the integral equation formulation for the electric potential as well as the accuracy of the spatial scheme. We solve the Laplace equation in the exterior and interior of an interface, shown in Figure 1, whose position vector $\mathbf{x}(u, v)$ is given by

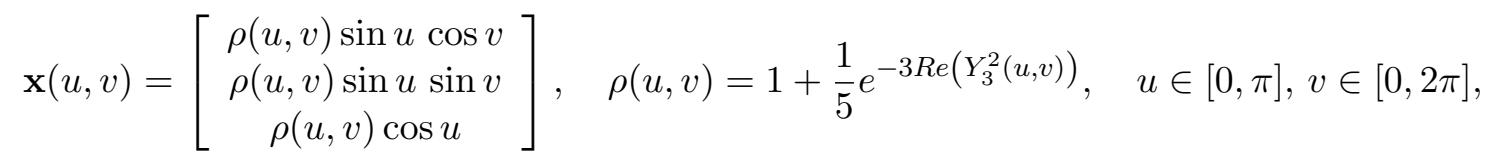

and subject to the following jump conditions at the interface:

$$
\llbracket \phi \rrbracket_{\gamma}=V_{m} \quad \text { and } \quad \llbracket \sigma \partial \phi / \partial \mathbf{n} \rrbracket_{\gamma}=J_{m} .
$$

As in Section 3, we assume that the potential is given by (7). The jump condition on the potential in (34) is satisfied by definition and applying the second boundary condition results in the following integral equation for the unknown function $q$,

$$
\left(\frac{1}{2}+\eta \mathcal{S}^{\prime}\right) q=\eta \mathcal{D}^{\prime}\left[V_{m}\right]+\frac{1}{\sigma_{i}+\sigma_{e}} J_{m}
$$

An analytic solution to this problem is constructed as follows: (i) place sources with arbitrary strengths randomly in the exterior and interior to $\gamma$, (ii) evaluate the jumps $V_{m}$ and $J_{m}$ corresponding to this source distribution, (iii) similarly, evaluate $q=\llbracket \partial \phi / \partial \mathbf{n} \rrbracket_{\gamma}$ and use it as an analytic solution to compare with the numerical solution of (35).

In Figure 1 (a) and (b), we sketch the jump conditions corresponding to our choice of source distribution and the table lists the errors in computing $q$ and $\mathbf{f}_{E}$ for increasing values of the spherical 
(a)

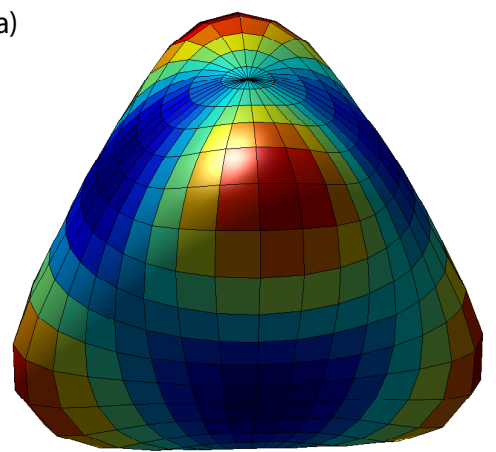

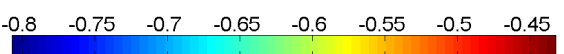

(b)

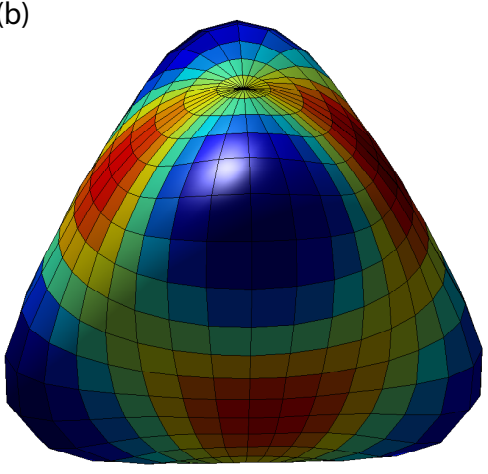

$\begin{array}{llllll}0.4 & 0.5 & 0.6 & 0.7 & 0.8 & 0.9\end{array}$

\begin{tabular}{ccccc}
\hline$p$ & $M$ & $\left\|q-q^{*}\right\|_{\infty}$ & $\left\|\mathbf{f}_{E}-\mathbf{f}_{E}^{*}\right\|_{\infty}$ & $N_{\text {iter }}$ \\
\hline 12 & 312 & $2.5 e-03$ & $2.7 e-02$ & 9 \\
16 & 544 & $3.0 e-04$ & $9.7 e-03$ & 9 \\
24 & 1200 & $3.7 e-05$ & $8.7 e-04$ & 9 \\
32 & 2112 & $2.3 e-06$ & $2.2 e-04$ & 9 \\
40 & 3280 & $3.7 e-07$ & $1.5 e-05$ & 9 \\
\hline
\end{tabular}

Figure 1: Relative errors in computing the jump in normal derivative $q$ and the electric force on the membrane $\mathbf{f}_{E}$ for the test case in Example 1 with $\sigma_{i}=2$ and $\sigma_{e}=1$. Here, $p$ is the spherical harmonic order and $M$ is the corresponding number of spatial discretization points. The boundary conditions (34) and corresponding analytic solution $\left(q^{*}, \mathbf{f}_{E}^{*}\right)$ are evaluated using the electric potential generated by randomly placed point sources away from the membrane. The corresponding jump conditions $V_{m}$ and $J_{m}$ in (34) are color mapped onto the given membrane shape in (a) and (b) respectively. The observable spectral convergence in the relative errors validates the integral equation formulation (35), the singular-integral evaluation $\mathcal{S}^{\prime}[q]$, and the hyper-singular integral evaluation $\mathcal{D}^{\prime}\left[V_{m}\right]$. The number of GMRES iterations in solving (35) are listed in the last column.

harmonic order, which validates the super-algebraic convergence rate of our method. The advantage of our indirect BIE formulation is that the integral operator $\left(\frac{1}{2}+\eta \mathcal{S}^{\prime}\right)$, which needs to be inverted, has a bounded condition number. From the number of GMRES required to solve (35) listed in Figure 1, it is clear that we get mesh-independent convergence ${ }^{1}$. Note that similar results are obtained for any other values of $\sigma_{i}$ and $\sigma_{e}$ since $|\eta|<1$.

Example 2 (Imposed electric field). Consider a vesicle subjected to an uniform electric field, $\mathbf{E}_{\infty}=(0,0,1)$, and in the absence any imposed flow. Suppose the permittivity and the viscosity ratios are unity, the conductivity ratio $\frac{\sigma_{i}}{\sigma_{e}}$ is less than one and the membrane conductance is nonzero. Under such conditions, the vesicle is known, through experiments, to reach an equilibrium with its shape transitioning through a "tube-like" phase [8, 9, 53]. In Figure 2, we show snapshots

\footnotetext{
${ }^{1}$ While this is the case for cases we have tested, it is conceivable that preconditioning will be necessary to achieve mesh-independent convergence for more complicated, close to self-touching geometries [35].
} 
from our three-dimensional simulation of this setting that illustrates similar transitionary behavior ${ }^{2}$. The initial shape is set to an $2-1$ ellipsoid, $\mathbf{x}(u, v)=(\sin u \cos v, \sin u \sin v, 2 \cos u)$.

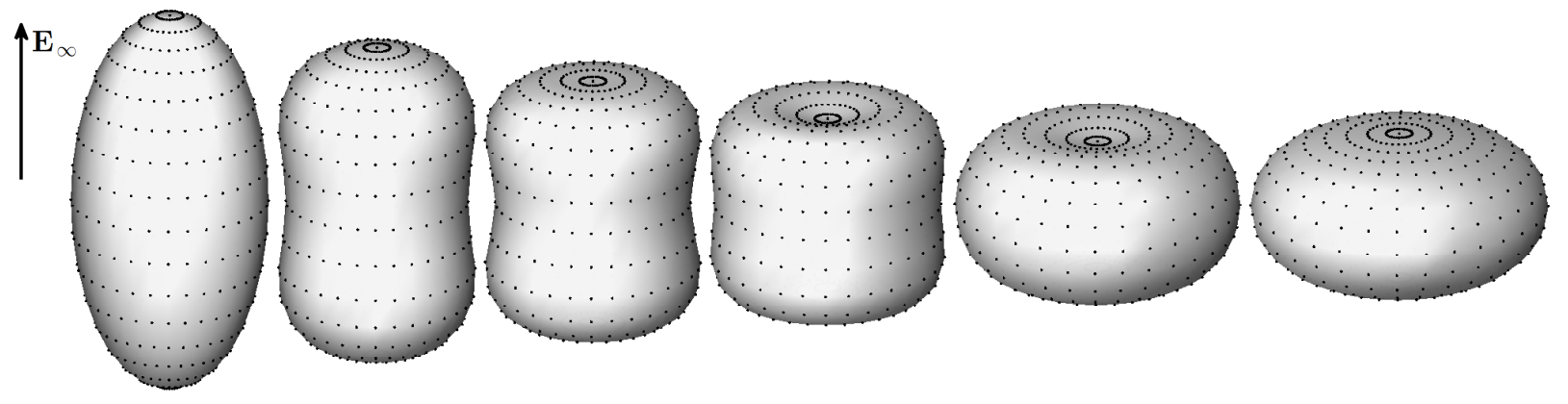

Figure 2: Snapshots from the EHD simulation of a single vesicle subjected to an external electric field $\mathbf{E}_{\infty}=(0,0,1)$ at time-steps $0,16 \triangle t, 24 \triangle t, 32 \triangle t, 40 \triangle t$ and $160 \triangle t$ respectively (from left to right) with $\triangle t=\frac{1}{2}$. The parameters for this example are given by $\sigma_{i}=1, \sigma_{e}=2, G_{m}=5, C_{m}=1, \kappa_{B}=0.1$ and no contrast in viscosity and permittivity of the exterior and interior fluids. The vesicle experiences compressional force due to the imposed electric field and undergoes prolate to oblate shape transformation transitioning through a tube-like phase that was observed experimentally $[8,9,53]$. The viscous, elastic and electric forces balance at the equilibrium oblate shape.

We found that, even though the final equilibrium shapes are the same, the intermediary shapes of the vesicle strongly depend on the electric capillary number, defined as the ratio of electric and elastic time scales [45],

$$
C a=\frac{t_{e l}}{t_{\kappa}}=\frac{\epsilon \mathbf{E}_{\infty}^{2}}{\kappa_{B}} \frac{3 V}{4 \pi},
$$

where $V$ is the volume enclosed by the vesicle. In Figure 3, we demonstrate the evolution of a vesicle with higher capillary number. Unlike the previous test case, tube-like phase is not observed in this simulation. The tension is uniformly higher for high $C a$ case as shown in Figure 4. Finally, note that if the conductivity of the interior fluid is higher compared to the exterior, the equilibrium shapes are in form of prolates as supported by previous theoretical predictions [33].

Example 3 (Imposed electric and flow fields). When a vesicle is subjected to linear shear flow, it undergoes tank-treading if there is no viscosity contrast. The angle of inclination at which the vesicle tank-treads dictates the effective viscosity of a dilute suspension at the macro-scale. For example, if it aligns with the background velocity profile, the vesicle presents less resistance to shear, thereby, the effective viscosity would be lower. In Figure 5, we consider three cases and compare the inclination angles. Clearly, applying the electric field alters the angle of inclination, consequently, the effective viscosity.

\footnotetext{
${ }^{2}$ Note that in experiments, generally, an AC field or a DC pulse is applied as opposed to the uniform electric field considered here. Therefore, the current results can be viewed as representative only for a short duration between pulses. In experiments, the vesicle goes through a prolate-oblate-prolate cycle.
} 


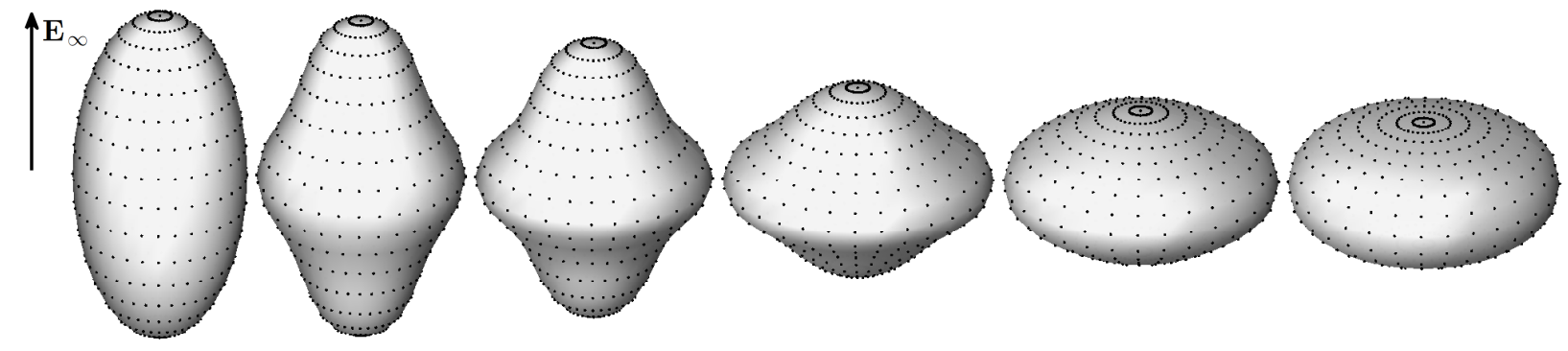

Figure 3: Snapshots from a similar EHD simulation as in Figure 3 with all parameters the same except the bending modulus is lower, $\kappa_{B}=0.01$. While the final equilibrium shape is the same, the transient shapes differ significantly (more contrasting details in Figure 4). The experimentally observed tube-like shapes can be replicated in numerical simulations only for lower values of Ca (e.g., Figure 2), that is, when the restoring bending time-scale is higher than the distorting electric time-scale.

\section{Conclusions}

We derived a set of integro-differential equations in this work that describe the coupled electro and hydrodynamics of a vesicle based on the leaky-dielectric model. The main advantage of the new formulation is that the linear systems arising from the BIEs are well-conditioned, allowing rapid solution via iterative methods. Derivatives, singular and hypersingular integrals are all computed with spectral accuracy via spherical harmonic representations. A semi-implicit time-stepping for evolving the membrane position allowed us to simulate vesicle EHD with modest number of timesteps (compared to explicit methods). Numerical experiments demonstrated the accuracy of our model and results from simulations of a vesicle in applied uniform electric field are consistent with previous theoretical predications.

There are a number of interesting questions in biomembrane mechanics one can answer with the code developed here - can vesicles/cells undergo self-locomotion by modulating their transmembrane potential [31]? What effect does reduced volume have on the morphological phase diagram [1]? These or other questions of practical importance will be the subject of future investigation. Furthermore, a more detailed analysis of electrorheology, the effect of viscosity and permittivity contrasts, time-varying imposed electric fields (DC pulses and AC fields) will be carried out in a future article. Another direction of interest is applying more general nonlinear models such as the Poisson-Boltzmann equation for the electric potential, essentially replacing the leaky dielectric fluids with solvent electrolytes.

\section{Acknowledgements}

This work was supported by the National Science Foundation under grants DMS-1224656 and DMS1418964, and by a Faculty Enhancement Award from ORAU. The author also acknowledges the computational resources and services provided by Advanced Research Computing at the University of Michigan, Ann Arbor. 

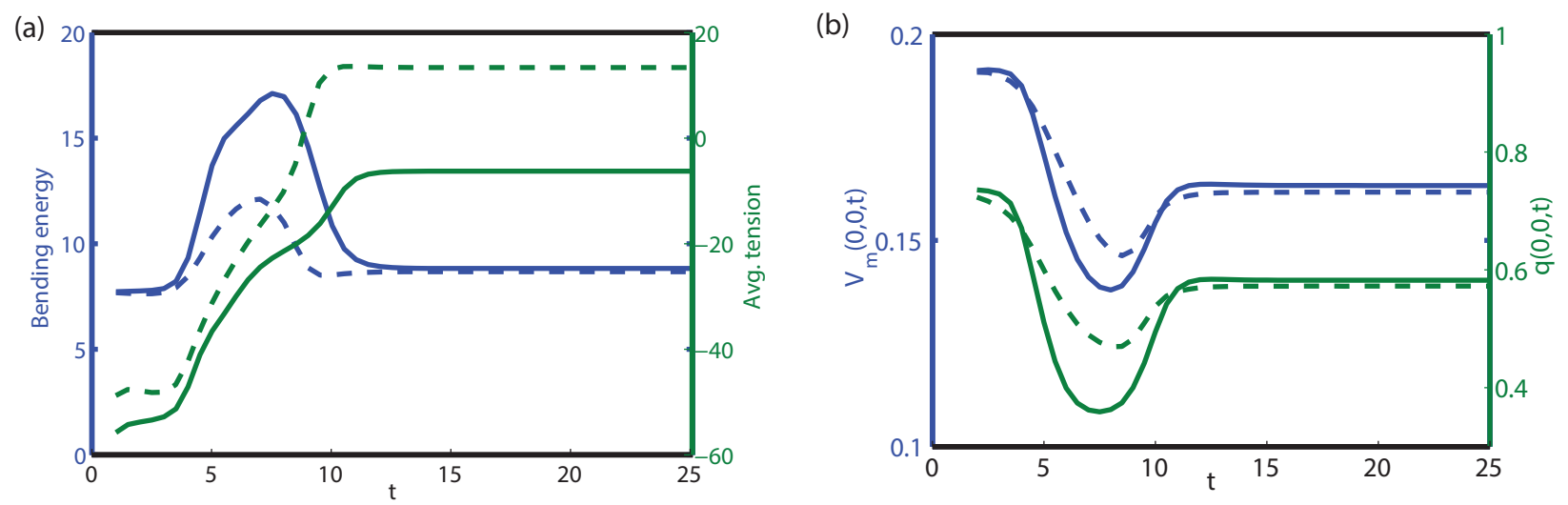

Figure 4: (a) Evolution of the scaled bending energy $\frac{1}{\kappa_{B}} \mathcal{E}$ (blue curves) and the averaged tension $\int_{\gamma} \sigma d \gamma$ (green curves). Solid curves correspond to $\kappa_{B}=0.01$ and dashed curves correspond to $\kappa_{B}=0.1$. The rest of the parameters are the same as in Figure 2. We make the following two observations for the lower $C a$ case compared to the higher: the membrane tension is uniformly higher; the intermediary shapes have lower bending energy although the final equilibrium shapes are the same. (b) Evolution of the transmembrane potential $V_{m}$ (blue curves) and the membrane charge density $q$ (green curves) measured at the north pole.

\section{References}

[1] S. Aranda, K. A. Riske, R. Lipowsky, And R. Dimova, Morphological transitions of vesicles induced by alternating electric fields, Biophysical journal, 95 (2008), pp. L19-L21.

[2] U. M. Ascher and L. R. Petzold, Computer Methods for Ordinary Differential Equations and Differential-Algebraic Equations, Society for Industrial and Applied Mathematics, Philadelphia, PA, USA, 1998.

[3] J. Beaucourt, F. Rioual, T. Séon, T. Biben, and C. Misbah, Steady to unsteady dynamics of a vesicle in a flow, Physical Review E, 69 (2004), p. 011906.

[4] T. Biben, A. Farutin, and C. Misbah, Three-dimensional vesicles under shear flow: Numerical study of dynamics and phase diagram, Physical Review E, 83 (2011), p. 031921.

[5] I. Cantat, K. Kassner, And C. Misbah, Vesicles in haptotaxis with hydrodynamical dissipation, The European Physical Journal E: Soft Matter and Biological Physics, 10 (2003), pp. 175-189.

[6] I. Cantat and C. Misbah, Dynamics and similarity laws for adhering vesicles in haptotaxis, Phys. Rev. Lett., 83 (1999), pp. 235-238.

[7] D. Dimitrov, Electroporation and electrofusion of membranes, Handbook of Biological Physics, 1 (1995), pp. 851-901.

[8] R. Dimova, N. Bezlyepkina, M. D. Jordo, R. L. Knorr, K. A. Riske, P. M. Staykova, M. Vlahovska, T. Yamamoto, P. Yang, and R. Lipowsky, Vesicles in electric fields: Some novel aspects of membrane behavior, Soft Matter, 5 (2009), pp. 3201 3212 . 

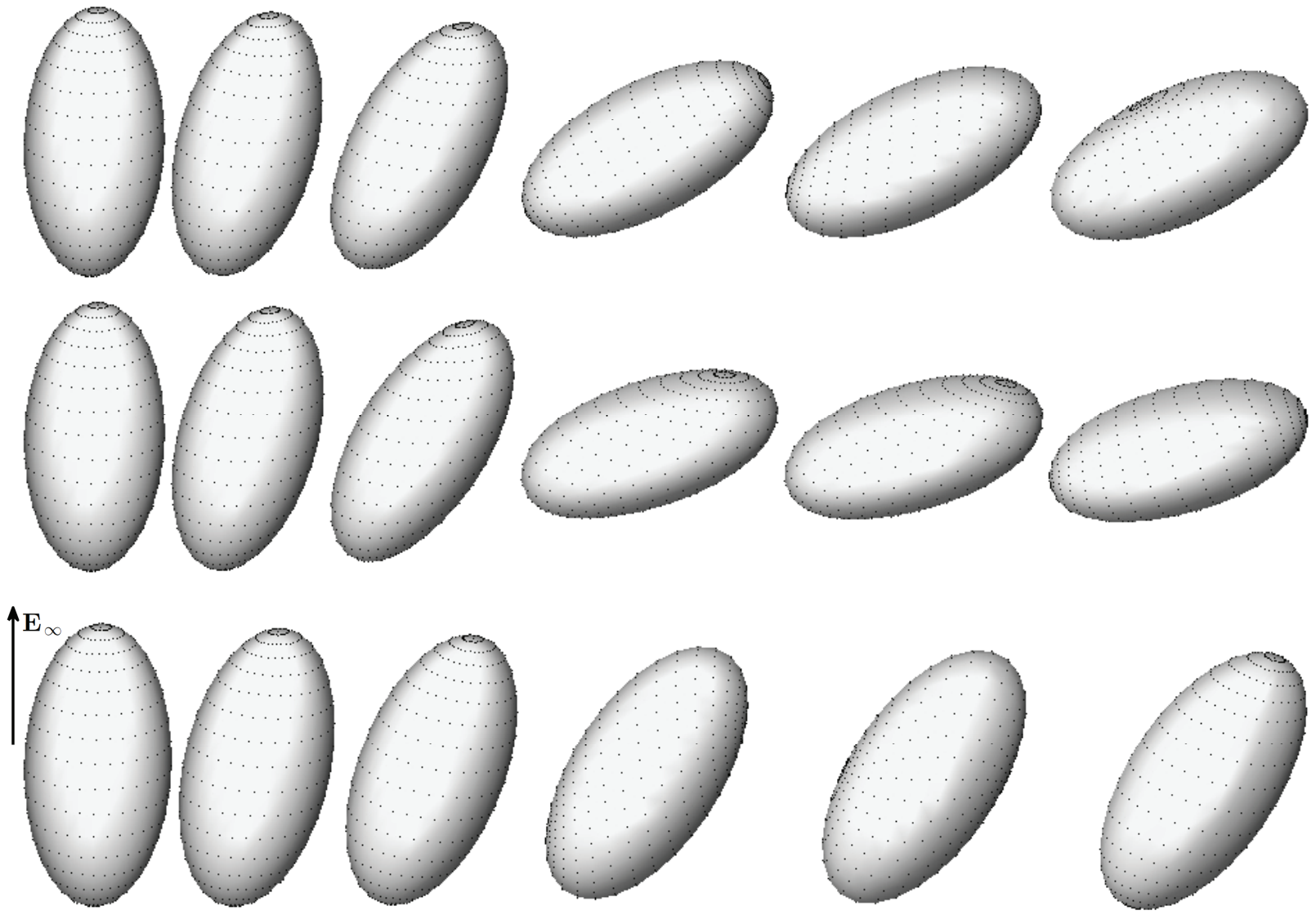

Figure 5: Snapshots of a vesicle subjected to linear shear flow (i) in the absence of electric field (top row), (ii) with $\mathbf{E}_{\infty}=(0,0,1), \sigma_{i}=1, \sigma_{e}=2$ (middle row), and (iii) with $\mathbf{E}_{\infty}=(0,0,1), \sigma_{i}=2, \sigma_{e}=1$ (bottom row). As expected, in the case where the interior-to-exterior conductivity ratio is less than one, the inclination angle (with $x$-axis) is lower since the resting shape is an oblate. The converse is true when the ratio is greater than one. This clearly demonstrates the effect of EHD on the rheology of dilute vesicle suspensions.

[9] R. Dimova, K. A. Riske, S. Aranda, N. Bezlyepkina, R. L. Knorr, and R. Lipowsky, Giant vesicles in electric fields, Soft Matter, 3 (2007), p. 817.

[10] A. Farutin, T. Biben, And C. Misbah, $3 d$ numerical simulations of vesicle and inextensible capsule dynamics, Journal of Computational Physics, 275 (2014), pp. 539-568.

[11] M. Ganesh And I. G. Graham, A high-order algorithm for obstacle scattering in three dimensions, Journal of Computational Physics, 198 (2004), pp. 211-242.

[12] Z. Gimbutas and S. Veerapaneni, A fast algorithm for spherical grid rotations and its application to singular quadrature, SIAM Journal on Scientific Computing, 35 (2013).

[13] M. Golzio, Direct visualization at the single-cell level of electrically mediated gene delivery, Proceedings of the National Academy of Sciences, 99 (2002), pp. 1292-1297. 
[14] I. G. Graham And I. H. Sloan, Fully discrete spectral boundary integral methods for helmholtz problems on smooth closed surfaces in r-3, Numerische Mathematik, 92 (2002), pp. 289-323.

[15] H.-D. HAN, The boundary element method for solving variational inequalities, Computational mathematics in China (Eds: Zhong-Ci Shi and Chung-Chun Yang), American Mathematical Society, 163 (1994), pp. 55-70.

[16] W. Helfrich, Elastic properties of lipid bilayers: theory and possible experiments., Zeitschrift für Naturforschung. Teil C: Biochemie, Biophysik, Biologie, Virologie, 28 (1973), p. 693.

[17] R. Heller, R. Gilbert, And M. Jaroszeski, Clinical applications of electrochemotherapy., Advanced drug delivery reviews, 35 (1999), pp. 119-129.

[18] Q. Hu, S. Viswanadham, R. Joshi, K. Schoenbach, S. Beebe, and P. Blackmore, Simulations of transient membrane behavior in cells subjected to a high-intensity ultrashort electric pulse, Physical Review E, 71 (2005), p. 031914.

[19] W.-F. Hu, M.-C. LAI, Y. Seol, And Y.-N. Young, Vesicle electrohydrodynamic simulations by coupling immersed boundary and immersed interface method, Journal of Computational Physics, 317 (2016), pp. 66-81.

[20] E. M. Kolahdouz And D. Salac, Electrohydrodynamics of three-dimensional vesicles: a numerical approach, SIAM Journal on Scientific Computing, 37, pp. B473-B494.

[21] E. M. Kolahdouz And D. Salac, Dynamics of three-dimensional vesicles in dc electric fields, Physical Review E, 92 (2015), p. 012302.

[22] P. Kolm And V. Rokhlin, Numerical quadratures for singular and hypersingular integrals, Computers \& Mathematics with Applications, 41 (2001), pp. 327-352.

[23] M. Kraus, W. Wintz, U. Seifert, and R. Lipowsky, Fluid vesicles in shear flow, Phys. Rev. Lett., 77 (1996), pp. 3685-3688.

[24] M. Kraus, W. Wintz, U. Seifert, And R. Lipowsky, Fluid vesicles in shear flow, Physical Review Letters, 77 (1996).

[25] R. KRESS, Linear Integral Equations, no. v. 82 in Applied Mathematical Sciences, Springer New York, 1999.

[26] G. Marple, A. Barnett, A. Gillman, and S. Veerapaneni, A fast algorithm for simulating multiphase flows through periodic geometries of arbitrary shape, arXiv preprint arXiv:1510.05616, (2015).

[27] L. C. McConnell, M. J. Miksis, and P. M. Vlahovska, Vesicle electrohydrodynamics in dc electric fields, IMA Journal of Applied Mathematics, (2013).

[28] — Continuum modeling of the electric-field-induced tension in deforming lipid vesicles, The Journal of chemical physics, 143 (2015), p. 243132. 
[29] L. C. McConnell, P. M. Vlahovska, and M. J. Miksis, Vesicle dynamics in uniform electric fields: squaring and breathing, Soft matter, 11 (2015), pp. 4840-4846.

[30] J. Melcher And G. TAYlor, Electrohydrodynamics: a review of the role of interfacial shear stresses, Annual Review of Fluid Mechanics, 1 (1969), pp. 111-146.

[31] J. L. Moran And J. D. Posner, Electrokinetic locomotion due to reaction-induced charge auto-electrophoresis, Journal of Fluid Mechanics, 680 (2011), pp. 31-66.

[32] E. Neumann, A. E. Sowers, And C. A. Jordan, Electroporation and electrofusion in cell biology, Springer, 1989.

[33] H. Nganguia and Y.-N. Young, Equilibrium electrodeformation of a spheroidal vesicle in an ac electric field, Physical Review E, 88 (2013), p. 052718.

[34] B. Quaife And G. Biros, High-volume fraction simulations of two-dimensional vesicle suspensions, Journal of Computational Physics, 274 (2014), pp. 245-267.

[35] — On preconditioners for the laplace double-layer in 2d, Numerical Linear Algebra with Applications, 22 (2015), pp. 101-122.

[36] A. Rahimian, S. K. Veerapaneni, and G. Biros, Dynamic simulation of locally inextensible vesicles suspended in an arbitrary two-dimensional domain, a boundary integral method, Journal of Computational Physics, 229 (2010), pp. 6466-6484.

[37] A. Rahimian, S. K. Veerapaneni, D. Zorin, and G. Biros, Boundary integral method for the flow of vesicles with viscosity contrast in three dimensions, Journal of Computational Physics, 298 (2015), pp. 766-786.

[38] K. A. Riske And R. Dimova, Electro-deformation and poration of giant vesicles viewed with high temporal resolution, Biophysical journal, 88 (2005), pp. 1143-1155.

[39] — Electric pulses induce cylindrical deformations on giant vesicles in salt solutions, Biophysical journal, 91 (2006), pp. 1778-1786.

[40] M. E. Rose, Elementary theory of angular momentum, Courier Dover Publications, 1995.

[41] Y. SAAD, Iterative Methods for Sparse Linear Systems, 2nd edition, SIAM, Philadelpha, PA, 2003.

[42] M. Sadik, J. Li, J. Shan, D. Shreiber, And H. Lin, Vesicle deformation and poration under strong dc electric fields, Physical Review E, 83 (2011).

[43] D. SAVILLE, Electrohydrodynamics: the taylor-melcher leaky dielectric model, Annual review of fluid mechanics, 29 (1997), pp. 27-64.

[44] J. T. Schwalbe, P. M. Vlahovska, and M. J. Miksis, Lipid membrane instability driven by capacitive charging, Physics of Fluids, 23 (2011), p. 04170.

[45] J. T. Schwalbe, P. M. Vlahovska, And M. J. Miksis, Vesicle electrohydrodynamics, Physical Review E, 83 (2011), p. 046309. 
[46] S. Sukumaran and U. Seifert, Influence of shear flow on vesicles near a wall: A numerical study, Physical Review E, 64 (2001).

[47] G. TAYLOR, Studies in electrohydrodynamics. $i$. the circulation produced in a drop by electrical field, in Proceedings of the Royal Society of London A: Mathematical, Physical and Engineering Sciences, vol. 291, The Royal Society, 1966, pp. 159-166.

[48] D. Van SwaAy ET AL., Microfluidic methods for forming liposomes, Lab on a Chip, 13 (2013), pp. $752-767$.

[49] S. K. Veerapaneni, D. Gueyffier, G. Biros, and D. Zorin, A numerical method for simulating the dynamics of $3 d$ axisymmetric vesicles suspended in viscous flows, Journal of Computational Physics, 228 (2009), pp. 7233-7249.

[50] S. K. Veerapaneni, D. Gueyffier, D. Zorin, and G. Biros, A boundary integral method for simulating the dynamics of inextensible vesicles suspended in a viscous fluid in 2D, Journal of Computational Physics, 228 (2009), pp. 2334-2353.

[51] S. K. Veerapaneni, A. Rahimian, G. Biros, and D. Zorin, A fast algorithm for simulating vesicle flows in three dimensions, J. Comput. Phys., 230 (2011), pp. 5610-5634.

[52] P. M. Vlahovska, Voltage-morphology coupling in biomimetic membranes: dynamics of giant vesicles in applied electric fields, Soft matter, 11 (2015), pp. 7232-7236.

[53] P. M. Vlahovska, R. S. Gracia, S. Aranda-Espinoza, and R. Dimova, Electrohydrodynamic model of vesicle deformation in alternating electric fields, Biophysical journal, 96 (2009), pp. 4789-4803.

[54] W. Ying And J. T. Beale, A fast accurate boundary integral method for potentials on closely packed cells, Communications in Computational Physics, 14 (2013), pp. 1073-1093.

[55] J. Zhang, J. D. ZAhn, W. TAN, AND H. Lin, A transient solution for vesicle electrodeformation and relaxation, Physics of Fluids (1994-present), 25 (2013), p. 071903.

[56] H. Zhao, A. H. G. Isfahani, L. N. Olson, and J. B. Freund, A spectral boundary integral method for flowing blood cells, J. Comput. Phys., 229 (2010), pp. 3726-3744. 